\title{
REARRANGEMENT INVARIANT NORMS OF SYMMETRIC SEQUENCE NORMS OF INDEPENDENT SEQUENCES OF RANDOM VARIABLES
}

\author{
STEPHEN MONTGOMERY-SMITH
}

\begin{abstract}
Let $X_{1}, X_{2}, \ldots, X_{n}$ be a sequence of independent random variables, let $M$ be a rearrangement invariant space on the underlying probability space, and let $N$ be a symmetric sequence space. This paper gives an approximate formula for the quantity \|\|$\left(X_{i}\right)\left\|_{N}\right\|_{M}$ whenever $L_{q}$ embeds into $M$ for some $1 \leq q<\infty$. This extends work of Johnson and Schechtman who tackled the case when $N=\ell_{p}$, and recent work of Gordon, Litvak, Schütt and Werner who obtained similar results for Orlicz spaces.
\end{abstract}

\section{INTRODUCTION}

In a recent paper [4], Gordon, Litvak, Schütt and Werner considered the problem of computing $\mathbb{E}\left\|\left(a_{i} \xi_{i}\right)\right\|_{N}$, where $N$ is an Orlicz sequence space, $a_{1}, a_{2}, \ldots, a_{n}$ are real numbers, and $\xi_{1}, \xi_{2}, \ldots, \xi_{n}$ are identically distributed random variables. They were able to construct an Orlicz function $\Lambda$ such that

$$
\mathbb{E}\left\|\left(a_{i} \xi_{i}\right)\right\|_{N} \approx\left\|\left(a_{i}\right)\right\|_{\Lambda},
$$

where $A \approx B$ means that the ratio of $A / B$ is bounded below and above by constants. Yehoram Gordon asked the question if a similar formula could be found for general independent random variables not just scalar multiples of identically distributed random variables.

1991 Mathematics Subject Classification. 60G50, 46B45, 46E30.

Key words and phrases. Independent random variables, symmetric sequence space, rearrangement invariant space.

The author was partially supported by NSF grant DMS 9870026, and a grant from the Research Office of the University of Missouri. 
Mark Rudelson asked about the possibility of computing higher moments.

Seeking to answer these questions, the author found it to his psychological advantage to consider the case of general rearrangement invariant spaces rather than just Orlicz norms. Let $M$ be a rearrangement invariant space on $[0,1]$, or equivalently on any probability space, and let $N$ be a symmetric sequence space. We will assume that all these vector spaces satisfy the triangle inequality. It seems quite likely that many of the formulae will extend to at least some quasi-Banach situations, but we do not explore this possibility here. We will normalize the spaces so that $\|1\|_{M}=\|(1,0, \ldots, 0)\|_{N}=1$. We seek to find an approximate formula for \|\|$\left(X_{i}\right)\left\|_{N}\right\|_{M}$.

The appropriate concept for describing our formula, the disjoint sum, or disjunctification, has been present in the literature for some time, for example, [2], [5], [7], [8]. This is the function on $[0, n]$ that takes $t$ to $X_{[t]+1}^{\#}(t-[t])$, where $[t]$ denotes the integer part of $t$, and $X_{i}^{\#}$ denotes the non-increasing rearrangment of $X_{i}$. We will write $Y$ for the non-increasing rearrangement of this function, that is, $Y:[0, n] \rightarrow[0, \infty]$ is a non-increasing function such that

$$
\text { measure }\{Y>t\}=\sum_{i=1}^{n} \operatorname{Pr}\left(X_{i}>t\right) \text {. }
$$

The conjecture we will explore is

$$
\|\|\left(X_{i}\right)\left\|_{N}\right\|_{M} \approx\left\|\left.Y\right|_{[0,1]}\right\|_{M}+\|(Y(i))\|_{N},
$$

where the constants of approximation may be allowed to depend upon M.

Indeed for all the special cases hitherto considered in the literature, this conjecture is true, as long as $M$ is far away from $L_{\infty}$. (It is clear that there must be some such restriction. For example, if $M=$ $L_{\infty}$ then \|\|$\left(X_{i}\right)\left\|_{N}\right\|_{M}=\left\|\left(\left\|X_{i}\right\|_{\infty}\right)\right\|_{N}$, and so equation (1) does not necessarily hold.) First, Rosenthal's inequality [13] can be interpreted as the truth of this statement in the cases that $N=\ell_{1}$ or $N=\ell_{2}$, and $M=L_{p}$ for $1 \leq p<\infty$. This was extended by Carothers and Dilworth [2] to the case when $M$ is a Lorentz space $L_{p, q}, 1 \leq p<\infty$, $1 \leq q \leq \infty$, and then by Johnson and Schechtman [8] to the case when $M$ is any rearrangement invariant space, even including the 
case when $M$ is a quasi-Banach space, as long as $L_{q}$ embeds into $M$, and $M$ embeds into $L_{r}$, via the natural embeddings, for some $0<r \leq q<\infty$. It is not hard to extend this last result to also allow $N=\ell_{p}$ for any $1 \leq p<\infty$.

The main result of this paper is the following.

Theorem 1. Equation (11) is true if there exists $1 \leq q<\infty$ such that $L_{q}$ embeds continuously via the natural embedding into $M$. In that case, the constants of approximation in equation (1) depend only upon $q$ and the constant of embedding.

In the last part of this paper, we will describe how to use this to recover some of the results of [4].

The author would like to express his sincere appreciation to Mark Rudelson for useful conversations, and for bringing this problem and reference [4] to his attention, and also to Joel Zinn for pointing out the reference [11].

\section{Proof of Main Theorem}

If $\left(x_{i}\right)$ is a sequence we will denote its non-increasing rearrangement by $\left(x_{i}^{*}\right)$. If $f$ is a function or random variable, we will denote its nonincreasing rearrangement by $f^{\#}$.

Lemma 2. Equation (1) is true if $M=L_{1}$ and $N=\ell_{\infty}$.

Proof. This follows because

$$
\frac{1}{2} \text { measure }\left\{\left.Y\right|_{[0,1]}>t\right\} \leq \operatorname{Pr}\left(\max _{i} X_{i}>t\right) \leq \operatorname{measure}\left\{\left.Y\right|_{[0,1]}>t\right\} .
$$

This has an elementary proof — see for example [5, Proposition 2.1] or [3]. Thus

$$
\|\|\left(X_{i}\right)\left\|_{\infty}\right\|_{1} \approx \int_{0}^{1} Y(t) d t .
$$

For each integer $1 \leq m \leq n$, let $k_{m}$ denote the sequence space $\left\|\left(x_{i}\right)\right\|_{k_{m}}=\sum_{i=1}^{m} x_{i}^{*}$.

Lemma 3. For each positive integer $m$, equation (1) is true if $M=$ $L_{1}$ and $N=k_{m}$, with constants of approximation independent of $m$. 
Proof. Let $I_{1}, I_{2}, \ldots, I_{n}$ be $\{0,1\}$-valued independent random variables that are also independent of $\left(X_{i}\right)$, where $\operatorname{Pr}\left(I_{i}=1\right)=1 / m$. Applying equation (3) to the sequence $\left(I_{i} X_{i}\right)$ we obtain

$$
\|\|\left(I_{i} X_{i}\right)\left\|_{\infty}\right\|_{1} \approx \int_{0}^{1} Y(m t) d t \approx \frac{1}{m}\left(\int_{0}^{1} Y(t) d t+\|\left(Y(i) \|_{k_{m}}\right) .\right.
$$

Next, let $\mathcal{M}$ denote the $\sigma$-field generated by $\left(X_{i}\right)$. Then applying equation (4), we see that

$$
\mathbb{E}\left(\left\|\left(I_{i} X_{i}\right)\right\|_{\infty} \mid \mathcal{M}\right) \approx \frac{1}{m}\left\|\left(X_{i}\right)\right\|_{k_{m}} .
$$

Thus we also obtain that

$$
\|\|\left(I_{i} X_{i}\right)\left\|_{\infty}\right\|_{1}=\left\|\mathbb{E}\left(\left\|\left(I_{i} X_{i}\right)\right\|_{\infty} \mid \mathcal{M}\right)\right\|_{1} \approx \frac{1}{m}\|\|\left(X_{i}\right)\left\|_{k_{m}}\right\|_{1} .
$$

The result follows.

Let $P$ denote the space of functions $f$ on $[0, n]$ for which its quasinorm

$$
\|f\|_{P}=\left\|\left.f^{\#}\right|_{[0,1]}\right\|_{M}+\left\|\left(f^{\#}(i)\right)\right\|_{N}
$$

is finite. In fact this quasi-norm is equivalent to a norm, viz, $\|f\|_{P^{\prime}}=$ $\left\|\left.f^{\#}\right|_{[0,1]}\right\|_{M}+\left\|\left(\int_{i-1}^{i} f^{\#}(t) d t\right)\right\|_{N}$ (see for example [12, Section 7]). However we will content ourselves with proving the following statement.

Lemma 4. For any function $f$ on $[0, n]$ we have $\|f(\cdot / 100)\|_{P} \leq$ $200\|f\|_{P}$.

Proof. First, since $M$ satisfies the triangle inequality, it follows that $\left\|\left.f(\cdot / 100){ }^{\#}\right|_{[0,1]}\right\|_{M} \leq 100\left\|\left.f^{\#}\right|_{[0,1 / 100]}\right\|_{M}$. Next, since $f^{\#}(i / 100) \leq$ $f^{\#}([i / 100])$, where $[t]$ denotes the integer part of $t$, we see that

$$
\begin{aligned}
\left\|\left(f^{\#}(i / 100)\right)\right\|_{N} & \leq 100\left\|\left(f^{\#}(i)\right)\right\|_{N}+\sum_{i=1}^{99} f^{\#}(i / 100) \\
& \leq 100\left\|\left(f^{\#}(i)\right)\right\|_{N}+100 \int_{0}^{1} f^{\#}(t) d t \\
& \leq 100\|f\|_{P} .
\end{aligned}
$$


Finally we need to cite a couple of results. For the case we need, $p=1$, the first result is essentially an immediate corollary of the Hoffmann-Jørgensen inequality [6], at least in the form found in [9, Proposition 1.3.2], and inequality (2). However we find an explicit reference to what we need in [5, Theorem 6.1]. The second result is [5, Theorem 7.1]. These concern maximal sums of vector valued random variables $U=\max _{k}\left\|\sum_{i=1}^{k} Z_{i}\right\|$, where $Z_{1}, Z_{2}, \ldots, Z_{n}$ are Banach-valued independent random variables. Let $V:[0,1] \rightarrow[0, \infty]$ be defined so that

$$
\text { measure }\{V>t\}=\min \left\{1, \sum_{i=1}^{n} \operatorname{Pr}\left(\left\|Z_{i}\right\|>t\right)\right\} \text {. }
$$

Theorem 5. If $p \geq 1$, then $\|U\|_{p} \approx U^{\#}\left(e^{-p} / 4\right)+\|V\|_{p}$.

Theorem 6. Suppose that $L_{q}$ embeds continuously into $M$ via the natural embedding, where $1 \leq q<\infty$. Then $\|U\|_{M} \approx\|U\|_{1}+\|V\|_{M}$, where the constant of approximation depends only upon $q$ and the embedding constant.

Proof of Theorem 1 . Let us first show the lower bound. Here the proof is very similar to the proof of [12, Theorem 27]. We know that

$$
\begin{aligned}
\left\|\left(x_{i}\right)\right\|_{N} & =\sup _{\|y\|_{N^{*} \leq 1}} \sum_{i=1}^{n} x_{i}^{*} y_{i}^{*} \\
& =\sup _{\|y\|_{N^{*} \leq 1}} \sum_{m=1}^{n}\left(y_{m}^{*}-y_{m+1}^{*}\right)\left\|\left(x_{i}\right)\right\|_{k_{m}},
\end{aligned}
$$

where by convention $y_{n+1}^{*}=0$, and $N^{*}$ denotes the dual space to $N$. From this, we immediately see that

$$
\begin{aligned}
\mathbb{E}\left\|\left(X_{i}\right)\right\|_{N} & \geq \sup _{\|y\|_{N^{*}} \leq 1} \sum_{m=1}^{n}\left(y_{m}^{*}-y_{m+1}^{*}\right) \mathbb{E}\left\|\left(X_{i}\right)\right\|_{k_{m}} \\
& \approx \sup _{\|y\|_{N^{*} \leq 1}} \sum_{m=1}^{n}\left(y_{m}^{*}-y_{m+1}^{*}\right)\left(\int_{0}^{1} Y(t) d t+\|(Y(i))\|_{k_{m}}\right) \\
& \approx \int_{0}^{1} Y(t) d t+\|(Y(i))\|_{N}
\end{aligned}
$$


since $y_{1}^{*} \leq 1$ whenever $\|y\|_{N^{*}} \leq 1$. To finish the lower bound, we see that

$$
2\|\|\left(X_{i}\right)\left\|_{N}\right\|_{M} \geq\|\|\left(X_{i}\right)\left\|_{\infty}\right\|_{M}+\mathbb{E}\left\|\left(X_{i}\right)\right\|_{N},
$$

and the result follows by equation (2).

Now let us focus on the upper bound. Really the first part of this proof follows by an inequality obtained independently by van Zuijlen [14], [15], [16], and Marcus and Pisier [10]. But we shall provide a self contained proof that is essentially a copy of the proof of this same result that may be found in [11, Theorem 5.1]. From Lemma 4, it follows that $\|Y(\cdot / 100)\|_{P} \leq 200\|Y\|_{P}$. We have that

$$
\begin{aligned}
\operatorname{Pr}\left(\left\|\left(X_{i}\right)\right\|_{N}>200\|Y\|_{P}\right) & \leq \operatorname{Pr}\left(\left\|\left(X_{i}\right)\right\|_{N}>\|Y(\cdot / 100)\|_{P}\right) \\
& \leq \operatorname{Pr}\left(\left\|\left(X_{i}\right)\right\|_{N}>\|(Y(i / 100))\|_{N}\right) \\
& \leq \operatorname{Pr}\left(\exists i: X_{i}^{*}>Y(i / 100)\right) \\
& \leq \sum_{i=1}^{n} \operatorname{Pr}\left(X_{i}^{*}>Y(i / 100)\right) \\
& \leq \sum_{i=1}^{n} \sum_{j_{1}<j_{2}<\cdots<j_{i}} \prod_{k=1}^{i} \operatorname{Pr}\left(X_{j_{k}}>Y(i / 100)\right) \\
& \leq \sum_{i=1}^{n} \frac{1}{i !}\left(\sum_{j=1}^{n} \operatorname{Pr}\left(X_{j}>Y(i / 100)\right)\right)^{i} \\
& \leq \sum_{i=1}^{n} \frac{i^{i}}{100^{i} i !} \\
& \leq \frac{1}{4 e}
\end{aligned}
$$

that is to say, $\left(\left\|\left(X_{i}\right)\right\|_{N}\right)^{\#}(1 / 4 e) \leq 200\|Y\|_{P}$.

Now we may apply Theorems 5 and 6 to $Z_{i}=X_{i} e_{i} \in N$, where $e_{i}$ denotes the $i$ th unit vector. In that case we see that $U=\left\|\left(X_{i}\right)\right\|_{N}$, and $V=\left.Y\right|_{[0,1]}$, and the result follows.

\section{Application to Orlicz Spaces}

In this section we will recover some of the results of Gordon, Litvak,

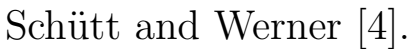


Lemma 7. Suppose that $M$ and $N$ are Orlicz spaces constructed from Orlicz functions $\Phi$ and $\Psi$ respectively. Define a function

$$
\Theta(x)= \begin{cases}\Psi(x) & \text { if } 0 \leq x \leq 1 \\ \Phi(x) & \text { if } x \geq 1\end{cases}
$$

Then $P$ is equivalent to the Orlicz space $L_{\Theta}$.

Proof. Note that because of the normalization on $M$ and $N$ that $\Phi(1)=\Psi(1)=1$. Also while $\Theta$ need not be an Orlicz function, it does satisfy the property that $\Theta(x) / x$ is an increasing function, and hence it is easily seen to be equivalent to the Orlicz function: $\tilde{\Theta}(x)=\int_{0}^{x} \frac{\Theta(t)}{t} d t$.

Suppose that $\|f\|_{L_{\Theta}} \leq 1$, that is $\int_{0}^{n} \Theta\left(f^{\#}(t)\right) d t \leq 1$. Then in particular $f^{\#}(1) \leq \int_{0}^{1} \Theta\left(f^{\#}(t)\right) d t \leq 1$. Thus

$$
\sum_{i=1}^{n} \Psi\left(f^{\#}(i)\right) \leq \Theta\left(f^{\#}(1)\right)+\int_{1}^{n} \Theta\left(f^{\#}(t)\right) d t \leq 2,
$$

and so $\sum_{i=1}^{n} \Psi\left(f^{\#}(i) / 2\right) \leq 1$, that is $\left\|\left(f^{\#}(i)\right)\right\|_{N} \leq 2$. Also, if $a=$ measure $\{f>1\}$ (so $a \leq 1)$, then

$$
\int_{0}^{1} \Phi\left(f^{\#}(t)\right) d t \leq \int_{0}^{a} \Theta\left(f^{\#}(t)\right) d t+(1-a) \leq 2,
$$

that is, $\left\|\left.f^{\#}\right|_{[0,1]}\right\|_{M} \leq 2$. Therefore $\|f\|_{P} \leq 4$.

Now suppose that $\|f\|_{P} \leq 1$. Again we see that $f^{\#}(1) \leq 1$, and $a=$ measure $\{f>1\} \leq 1$. Hence

$$
\int_{0}^{n} \Theta\left(f^{\#}(t)\right) d t \leq \int_{0}^{a} \Phi\left(f^{\#}(t)\right) d t+(1-a)+\sum_{i=1}^{n} \Psi\left(f^{\#}(i)\right) \leq 3 .
$$

Since $\Theta(x / 3) \leq \Theta(x) / 3$, it follows that $\|f\|_{L_{\Theta}} \leq 3$.

Now we will give a formulation of one of the results of [1], that gives a formula in the case that $M$ and $N$ are Orlicz spaces. While the formula presented here may appear superficially different than the formula given in [4], a short argument shows that it is equivalent (at least in the case discussed in [4], that is, when $M=L_{1}$ ). 
Theorem 8. Suppose that $\xi_{1}, \xi_{2}, \ldots, \xi_{n}$ are identically distributed random variables, and that $M$ and $N$ are Orlicz spaces where $M$ is constructed from an Orlicz function $\Phi$ satisfying

$$
\limsup _{x \rightarrow \infty} \log \Phi(x) / \log (x)<\infty .
$$

Then there exists an Orlicz function $\Lambda$, equivalent to the function $x \mapsto \mathbb{E}\left(\Theta\left(x \xi_{1}\right)\right)$, where $\Theta$ is the function constructed in Lemma $\square$, such that for all real numbers $a_{1}, a_{2}, \ldots, a_{n}$ we have

$$
\|\|\left(a_{i} \xi_{i}\right)\left\|_{N}\right\|_{M} \approx\left\|\left(a_{i}\right)\right\|_{L_{\Lambda}} \text {. }
$$

Proof. The condition on $\Phi$ tells us that there exists positive constants $c$ and $q$ such that $\Phi(x) \leq c x^{q}$ for sufficiently large $x$, that is, $L_{q}$ embeds into $M$. Thus by Theorem [1 and Lemma 7 we see that

$$
\|\|\left(a_{i} \xi_{i}\right)\left\|_{N}\right\|_{M} \approx\|Y\|_{\Theta} \text {. }
$$

But

$$
\|Y\|_{\Theta}=\inf \left\{\lambda>0: \sum_{i=1}^{n} \mathbb{E}\left(\Theta\left(a_{i} \xi_{i} / \lambda\right)\right) \leq 1\right\}=\left\|\left(a_{i}\right)\right\|_{\Lambda}
$$

Now we will give another proof of the following result that appears in [4. This paper also gives many other examples like this that are interesting.

Corollary 9. Suppose that $\gamma_{1}, \gamma_{2}, \ldots, \gamma_{n}$ are identically distributed normalized Gaussian random variables, and let $1 \leq m \leq n$ be an integer. Let $\Lambda$ be an Orlicz function equivalent to $x e^{-1 /(m x)^{2}}$. Then for all real numbers $a_{1}, a_{2}, \ldots, a_{n}$ we have

$$
\|\|\left(a_{i} \gamma_{i}\right)\left\|_{k_{m}}\right\|_{1} \approx\left\|\left(a_{i}\right)\right\|_{L_{\Lambda}} \approx \sum_{i=1}^{m} a_{i}^{*}+m \sup _{1 \leq i \leq n / m} a_{m i}^{*} \sqrt{1+\log i}
$$

Proof. An easy argument shows that if $M=L_{1}$ and $N=k_{m}$, then the Orlicz function $\Theta$ constructed in Lemma $\square$ is equivalent to the function $x \mapsto(x-1 / m)^{+}$. Then the function $\Lambda(x)$ constructed in Theorem 8 is equivalent to

$$
\sqrt{\frac{2}{\pi}} \int_{0}^{\infty}(x t-1 / m)^{+} e^{-t^{2} / 2} d t=x \sqrt{\frac{2}{\pi}} \int_{0}^{\infty}(t-1 / m x)^{+} e^{-t^{2} / 2} d t
$$


and the rest of the left approximation of (5) follows by simple calculations.

To see the second approximation of (5), note that for $0 \leq x \leq$ $m$ that $\Lambda(x)$ is equivalent to $x$, and that for $x>m$ that $\Lambda(x)$ is equivalent to $m e^{1-1 /(m x)^{2}}$. Then by an argument similar to the proof of Lemma 7, we see that

$$
\left\|\left(a_{i}\right)\right\|_{L_{\Lambda}} \approx\left\|\left(a_{i}^{*}\right)_{1 \leq i \leq m}\right\|_{1}+m\left\|\left(a_{m i}^{*}\right)\right\|_{L_{\exp \left(1-1 / x^{2}\right)}} .
$$

Finally we need to show that

$$
\left\|\left(b_{i}^{*}\right)\right\|_{L_{\exp \left(1-1 / x^{2}\right)}} \approx \sup _{i} b_{i}^{*} \sqrt{1+\log i} .
$$

This is essentially a sequential version of results from [1]. Suppose

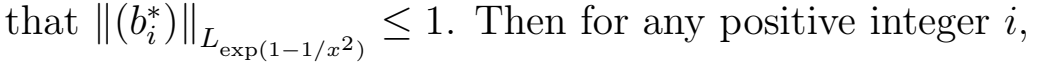

$$
i \exp \left(1-1 /\left(b_{i}^{*}\right)^{2}\right) \leq \sum_{j} \exp \left(1-1 /\left(b_{j}^{*}\right)^{2}\right) \leq 1,
$$

that is, $b_{i}^{*} \sqrt{1+\log i} \leq 1$. Conversely, if $\sup _{i} b_{i}^{*} \sqrt{1+\log i} \leq 1$, then

$$
\sum_{i} \exp \left(1-1 /\left(b_{i}^{*} / 2\right)^{2}\right) \leq \sum_{i} \exp (-3-4 \log i)=e^{-3} \sum_{i} \frac{1}{i^{4}} \leq 1,
$$

that is, $\left\|\left(b_{i}^{*}\right)\right\|_{L_{\exp \left(1-1 / x^{2}\right)}} \leq 2$.

We remark that a similar argument shows that if $\xi_{1}, \xi_{2}, \ldots, \xi_{n}$ are identically distributed random variables with $E\left|\xi_{1}\right|=1$, then

$$
\|\|\left(a_{i} \xi_{i}\right)\left\|_{k_{m}}\right\|_{1} \approx \sum_{i=1}^{m} a_{i}^{*}+m\|\|\left(a_{m i}^{*} \xi_{i}\right)\left\|_{\infty}\right\|_{1} .
$$

Finally let us finish with another remark. In [1], the authors showed in the case that $M=L_{1}$ that their upper bound held even if the random variables were not independent. This can also hold in our more general case. In [12 was introduced the concept of what it means for a rearrangement invariant space to be $D^{*}$-convex. This property is held, for example, by all Orlicz spaces. Following the proof of [12, Theorem 27], it can be shown that equation (II) holds even if the sequence $\left(X_{i}\right)$ is not necessarily independent, as long as $M=L_{1}$ and $P$ is $D^{*}$-convex. It is easy to see from the definition that 
the condition that $P$ be $D^{*}$-convex cannot be dropped. We leave the details to the interested reader.

\section{REFERENCES}

[1] Bennett C.; Rudnick K. On Lorentz-Zygmund spaces, Dissert. Math. 175 (1980), 1-72.

[2] Carothers, N.L.; Dilworth, S.J. Inequalities for sums of independent random variables, Proc. Amer. Math. Soc. 104 (1988), 221-226.

[3] Giné, E.; Zinn, J. Central limit theorems and weak laws of large numbers in certain Banach spaces. Z. Wahrsch. Verw. Gebiete, 62 (1983), 323-354.

[4] Gordon, Y.; Litvak, A.; Schütt, C.; Werner, E. Orlicz norms of sequences of random variables, preprint.

[5] Hitczenko P.; Montgomery-Smith S. Measuring the magnitude of sums of independent random variables, Annals of Probab. 29 (2001), 447-466.

[6] Hoffmann-Jørgensen, J. Sums of independent Banach space valued random variables. Studia Math. 52, (1974), 159-186.

[7] Johnson, W.B.; Maurey, B.; Schechtman, G.; Tzafriri, L. Symmetric structures in Banach spaces, Mem. Amer. Math. Soc. 217 (1979).

[8] Johnson, W.B.; Schechtman, G. Sums of independent random variables in rearrangement invariant function spaces, Ann. Probab. 17 (1989), 789-808.

[9] Kwapień, S.; Woyczyński, W.A. Random Series and Stochastic Integrals. Single and Multiple, Birkhäuser, Boston, (1992).

[10] Marcus, M.B.; Pisier, G. Characterizations of almost surely continuous $p$ stable random Fourier series and strongly stationary processes. Acta Math. 152 (1984), 245-301.

[11] Marcus, M.B.; Zinn, J. The bounded law of the iterated logarithm for the weighted empirical distribution process in the non-i.i.d. case. Ann. Probab. 12 (1984), 335-360.

[12] Montgomery-Smith, S.; Semenov, E. Random rearrangements and operators, Voronezh Winter Mathematical Schools, 157-183, Amer. Math. Soc. Transl. Ser. 2, 184, Amer. Math. Soc., Providence, RI, 1998.

[13] Rosenthal, H.P. On the subspaces of $L_{p}(p>2)$ spanned by sequences of independent symmetric random variables, Israel J. Math. 8 (1970), 273303.

[14] van Zuijlen, M.C.A. Some properties of the empirical distribution function in the non-i.i.d. case. Ann. Statisitics 4, (1976), 406-408.

[15] van Zuijlen, M.C.A. Properties of the empirical distribution function for independent nonidentically distributed random variables. Ann. Probability 6, (1978), 250-266.

[16] van Zuijlen, M.C.A. Properties of the empirical distribution function for independent nonidentically distributed random vectors. Ann. Probab. 10 (1982), 108-123. 
Department of Mathematics, University of Missouri, Columbia, MO 65211

E-mail address: stephen@math.missouri.edu

$U R L:$ http://www.math.missouri.edu/ ${ }^{\text {stephen }}$ 